\title{
Necessary and Sufficient Conditions for Current Conservation in the Framework of Relativistic Quantum Field Theory
}

\author{
Eldad Gal-Ezer \\ Dept. of Physics \& Astronomy, Tel-Aviv University, Ramat Aviv, Tel-Aviv, Israel \\ Received May 8, 1972; in revised form September 25, 1972
}

\begin{abstract}
Necessary and sufficient conditions for the conservation of vector currents covariant under translations and of the Conformal currents, are formulated and proven. Only the weak spectrum condition is assumed, thus the theorems serve as generalizations of a well-known theorem due to Coleman. This is done under the axioms of Relativistic Quantum Field Theory due to Wightman.
\end{abstract}

\section{Introduction}

In a previous publication [1], necessary and sufficient conditions for conservation of internal and conformal currents were formulated and proven. This treatment lacks rigour (e.g., distributions were integrated as ordinary functions). In the present treatment appropriate formulations and proofs are given in the framework of Relativistic Quantum Field Theory [2].

The theorems proven in this note serve as a rigorous formulation of the connection between current conservation and the fact that the corresponding charge annihilates (in some well defined manner) the vacuum state. This connection is known as "Coleman's Theorem" [3]. Our statements differ from previous versions of Coleman's Theorem as they are in a form of necessary and sufficient conditions and we do not demand a mass gap.

Thus, our theorems apply to cases where the presence of zero mass particles is an intrinsic feature of the symmetry group under consideration, as is the case with unbroken Chiral symmetry in the realization where the baryons are massless and unbroken Conformal symmetry where all the masses vanish. Furthermore, the theorems apply also to cases of spontanously broken internal and Conformal symmetries.

\section{The Framework and Praeliminaria}

The framework is Relativistic Quantum Field Theory. The postulates of the theory are given in Chap. 3 of Ref. 2. We stress that only the so 
called "weak spectrum condition" is assumed. This means that we do not demand a gap above the point $p=0$ (which corresponds to the vacuum state) in the energy momentum spectrum. Several specific assumptions which are used in the following will now be specified.

We assume the existence of four field operators, which we denote by $j^{\mu}(x)(\mu=0,1,2,3)$, whose transformation properties under the Poincare group will be given later. $j^{\mu}(x)$ are assumed to be relatively local and local with respect to the algebra $\mathscr{R}$ of localized operators under which the vacuum is cyclic. For every test function $f \in \mathscr{S}\left(R^{4}\right)$ (i.e., functions over the four dimensional real space with an infinite number of continuous derivatives and with strong decrease, i.e. faster than any inverse of a polynomial) $j^{\mu}(f)$ is an operator in the Hilbert space of states, having as a domain the common dense domain of the smeared field operators. Actually, the demand that $j^{\mu}(x)$ is tempered (i.e., $j^{\mu}(f)$ is defined for every $f \in \mathscr{S})$ is stronger than necessary for our purpose and we can equally do with the assumption that $j^{\mu}(f)$ is defined for every $f \in \mathscr{D}\left(R^{4}\right)$ (i.e., functions over the four dimensional real space with an infinite number of continuous derivatives and with compact support). This is true because we are dealing with Borcher's positive tensor distributions (Eq. (11)) and the class of Borcher's positive tensor distributions is identical to the class of Borcher's positive tempered tensor distributions [4].

We will use the common notation

$$
j^{\mu}(f)=\int d x f(x) j^{\mu}(x) .
$$

Let $\Theta_{r}(x)$ denote a $\mathscr{D}\left(R^{3}\right)$ function which satisfies

$$
\Theta_{r}(x)=\Theta\left(\frac{\boldsymbol{x}}{r}\right)= \begin{cases}1 & \frac{|\boldsymbol{x}|}{r}<1 \\ \alpha\left(\frac{|\boldsymbol{x}|}{r}\right) & 1 \leqq \frac{|\boldsymbol{x}|}{r} \leqq 2 \\ 0 & \frac{|\boldsymbol{x}|}{r}>2\end{cases}
$$

and let $\eta\left(x^{0}\right)$ be any $\mathscr{D}(R)$ function.

Let us assume that the current $j^{\mu}(x)$ is covariant under translations

$$
U(a) j^{\mu}(x) U^{-1}(a)=j^{\mu}(x+a)
$$

where $U(a)$ is a unitary representation of the translation group. 
Then, due to the invariance of the vacuum state $|0\rangle$ under translations

$$
\left\langle 0\left|\left[j^{0}(x), j^{0}(y)\right]\right| 0\right\rangle=W(x-y)
$$

is a distribution in the variable $x-y^{1}$.

Thus

$$
\left\langle 0\left|\left[j^{0}\left(\Theta_{r} \eta\right), j^{0}(y)\right]\right| 0\right\rangle=\int d x \Theta_{r}(x) \eta\left(x^{0}\right) W(x-y)
$$

is a $C^{\infty}\left(R^{4}\right)$ function of $y^{2}$ (i.e., it has an infinite number of continuous derivatives) and we can consider its value at a point.

In the following, we will be concerned with currents of the form [5]

$$
d^{\mu}(x)=x_{v} \theta^{\mu \nu} ; \mathscr{K}^{\mu \nu}(x)=\left(2 x^{v} x_{\lambda}-\delta_{\lambda}^{v} x^{2}\right) \theta^{\mu \lambda}(x)
$$

where the tensor $\theta^{\mu \nu}(x)$ is covariant under translations. In this case also, the following expressions are $C^{\infty}\left(R^{4}\right)$ functions of $y$ :

$$
\left\langle 0\left|\left[d^{0}\left(\Theta_{r} \eta\right), \theta^{\mu v}(y)\right]\right| 0\right\rangle ; \quad\left\langle 0\left|\left[\mathscr{K}^{0 v}\left(\Theta_{r} \eta\right), \theta^{\mu v}(y)\right]\right| 0\right\rangle
$$

and we can consider their values at a point.

\section{Two Theorems}

Theorem 1. Let $j^{\mu}(x)$ be a vector current covariant under the Poincaré group

$$
U(a, \Lambda) j^{\mu}(x) U^{-1}(a, \Lambda)=\left(\Lambda^{-1}\right)^{\mu}{ }_{v} j^{v}(\Lambda x+a)
$$

where $U(a, \Lambda)$ is a unitary representation of the Poincare group.

Then, the following four conditions are equivalent:

(i) $\partial_{\mu} j^{\mu}(y)=0$.

(ii) $\lim _{r \rightarrow \infty}\left\langle 0\left|\left[j^{0}\left(\Theta_{r} \eta\right), j^{0}(y)\right]\right| 0\right\rangle=0$

for every $\eta \in \mathscr{D}(R)$ and one arbitrary $y \in R^{4}$.

(iii) $\lim _{r \rightarrow \infty}\left\langle 0\left|\left[j^{0}\left(\Theta_{r} \eta\right), \partial_{0} j^{0}(y)\right]\right| 0\right\rangle=0$

for every $\eta \in \mathscr{D}(R)$ and one arbitrary $y \in R^{4}$.

(iv) $\lim _{r \rightarrow \infty}\left\langle 0\left|\left[j^{0}\left(\Theta_{r} \eta\right), \partial_{\mu} j^{\mu}(y)\right]\right| 0\right\rangle=0$

for every $\eta \in \mathscr{D}(R)$ and one arbitrary $y \in R^{4}$.

Remarks. 1) For simplicity of notation, we assumed $j^{\mu}(x)$ to be hermitian. Otherwise, we should carry a dagger sign over one current.

2) All the limits in (ii) to (iv) exist as, due to locality, they are achieved for finite $r_{0}=r_{0}(\eta, y)$.

3) The theorem remains valid if we change everywhere $\left[j^{0}\left(\Theta_{r} \eta\right), j^{0}(y)\right]$ to $\left[j^{0}\left(\Theta_{r}, y^{0}\right), j^{0}(\boldsymbol{y}, \eta)\right]$ etc. This may be more apealing, as there is no time smearing in the "charge" $j^{0}\left(\Theta_{r} y_{0}\right)$.

\footnotetext{
${ }^{1}$ See Theorem 3.2 in Ref. 2

${ }^{2}$ See page 42 in Ref. 2.
} 
Theorem 2. Let us denote

$$
d^{\mu}(x)=x_{v} \theta^{\mu \nu}(x) ; \quad \mathscr{K}^{\mu \nu}(x)=\left(2 x^{v} x_{\lambda}-x_{\lambda}^{v}\right) \theta^{\mu \lambda}(x)
$$

and let us assume that the hermitian tensor field $\theta^{\mu v}(x)$ satisfies

$$
\begin{gathered}
\theta^{\mu \nu}(x)=\theta^{v \mu}(x) ; \quad \partial_{\mu} \theta^{\mu v}(x)=0 ; \quad\left\langle 0\left|\theta^{\mu v}(x)\right| 0\right\rangle=0 \\
U(a, \Lambda) \theta^{\mu \nu}(x) U^{-1}(a, \Lambda)=\left(\Lambda^{-1}\right)^{\mu}{ }_{\sigma}\left(\Lambda^{-1}\right)^{v}{ }_{\kappa} \theta^{\sigma \kappa}(\Lambda x+a) .
\end{gathered}
$$

Then the following five conditions are equivalent:

(a) $\partial_{\mu} d^{\mu}(y)=\theta_{\mu}{ }^{\mu}(y)=0$.

(b) $\partial_{\mu} \mathscr{K}^{\mu v}(y)=2 y^{v} \theta_{\mu}^{\mu}(y)=0$.

(c) $\lim _{r \rightarrow \infty}\left\langle 0\left|\left[d^{0}\left(\Theta_{r} \eta\right), \theta_{\mu}{ }^{\mu}(y)\right]\right| 0\right\rangle=0$

for every $\eta \in \mathscr{D}(R)$ and one arbitrary $y \in R^{4}$.

(d) $\lim _{r \rightarrow \infty}\left\langle 0\left|\left[\mathscr{K}^{00}\left(\Theta_{r} \eta\right), \theta_{\mu}{ }^{\mu}(y)\right]\right| 0\right\rangle=0$ for every $\eta \in \mathscr{D}(R)$, one arbitrary $y \in R^{3}$ and every $y^{0} \in R$.

(e) $\lim _{r \rightarrow \infty}\left\langle 0\left|\left[\mathscr{K}^{0 i}\left(\Theta_{r} \eta\right), \theta_{\mu}{ }^{\mu}(y)\right]\right| 0\right\rangle=0$ for every $\eta \in \mathscr{D}(R)$, one $i=1,2,3$ and one arbitrary $y \in R^{4}$ with $y^{i} \neq 0$.

Remark. Similar to Remark 3 (after Theorem 1), this theorem remains valid if one removes the time smearing and puts it in $\theta_{\mu}{ }^{\mu}(y)$ in (c)-(e). Thus, in (c) we would have $\left[d^{0}\left(\Theta_{r} y_{0}\right), \theta_{\mu}{ }^{\mu}(y, \eta)\right]$ etc. In (d) it is then sufficient to demand the vanishing for $y^{0}=0$ only.

We will prove now Theorem 1.

We use a rigorous version of the Källen-Lehman representation [6], as given by Wightman and Gårding and by Reeh [7]

$$
\begin{gathered}
\left\langle 0\left|j^{\mu}(x) j^{v}(y)\right| 0\right\rangle=\int d p e^{-i p(x-y)} \varrho^{\mu v}(p) \\
\varrho^{\mu v}(p)=p^{\mu} p^{v} \varrho_{1}(p)+g^{\mu v} \varrho_{2}(p)
\end{gathered}
$$

where $\varrho_{1}(p)$ and $\varrho_{2}(p)$ are tempered measures with support in the close forward light cone $\bar{V}_{+}$, and are invariant under the restricted Lorentz group $\mathscr{L}_{+}^{\uparrow}$. Let us define

$$
\varrho(p)=p^{2} \varrho_{1}(p)+\varrho_{2}(p) .
$$

$\varrho(p)$ is also a Lorentz invariant tempered measure with support in $\bar{V}_{+}$. The general form of such a measure is [9]

$$
\varrho(p) d p=c \delta(p) d p+\int d \chi \varrho(\chi) \delta\left(p^{2}-\chi\right) d p ; \quad p_{0} \geqq 0
$$

where $\hat{\varrho}(\chi)$ is a tempered measure whose support is in the non-negative real line. 
Thus, for every $g \in \mathscr{S}\left(R^{4}\right)$ we have

$$
\int d p g(p) \varrho(p)=\int_{0}^{\infty} d \chi h(\chi) \varrho(\chi)+c g(0)
$$

where

$$
h(\chi)=\int_{p_{0} \geqq 0} d p g(p) \delta\left(p^{2}-\chi\right) .
$$

A useful result is [8] that for every $g(p) \in \mathscr{S}\left(R^{4}\right)$ whose support excludes an open region which contains the point $p=0$, the corresponding $h(\chi)$ is in $\mathscr{S}(R)$.

The equivalence of conditions (i) and (ii) of Theorem 1 is a consequence of the following two lemmas.

Lemma 1. The following two conditions are equivalent:

(i) $\partial_{\mu} j^{\mu}(y)=0$.

(ii) $p^{\mu} \varrho(p)=0$.

Lemma 2. The following two conditions are equivalent:

(i) $\lim _{r \rightarrow \infty}\left\langle 0\left|\left[j^{0}\left(\Theta_{r} \eta\right), j^{0}(y)\right]\right| 0\right\rangle=0$

$$
\text { for every } \eta \in \mathscr{D}(R) \text { and one arbitrary } y \in R^{4} \text {. }
$$

(ii) $p^{\mu} \varrho(p)=0$.

The equivalence of condition (i) of Theorem 1 to conditions (iii) and (iv) follows along the same line (i.e., from the equivalence of conditions (iii) and (iv) to $p^{\mu} \varrho(p)=0$ ).

Proof of Lemma 1. Let us assume that $p^{\mu} \varrho(p)=0$. According to Eqs. (11), (12) and (13) we have

$$
\left\langle 0\left|\partial_{\mu} j^{\mu}(x) \partial_{v} j^{\nu}(y)\right| 0\right\rangle=\int d p e^{-i p(x-y)} p_{\mu} p^{\mu} \varrho(p)=0 .
$$

Thus, for every $f \in \mathscr{D}\left(R^{4}\right)$ we have

$$
\partial_{\mu} j^{\mu}(f)|0\rangle=0
$$

and thus (see Theorem 4.3 in Ref. 2)

or

$$
\partial_{\mu} j^{\mu}(f)=0 \text { for every } f \in \mathscr{D}
$$

$$
\partial_{\mu} j^{\mu}(x)=0 \text {. }
$$

Let us assume now the Eq. (18) holds. Eqs. (11), (12) and (13) yield then

or

$$
0=\left\langle 0\left|j^{\mu}(x) \partial_{v} j^{\nu}(y)\right| 0\right\rangle=i \int d p e^{-i p(x-y)} p^{\mu} \varrho(p)
$$

$$
p^{\mu} \varrho(p)=0 .
$$

This concludes the proof of Lemma 1. 
Proof of Lemma 2. Let us first find an expression for the vacuum matrix element in terms of the spectral functions $\varrho(p)$ and $\varrho_{1}(p)$.

Defining the Fourier transforms

$$
\tilde{\Theta}_{r}(\boldsymbol{p})=\tilde{\Theta}_{\boldsymbol{r}}(-\boldsymbol{p})=\int d \boldsymbol{x} e^{-i \boldsymbol{p} \cdot \boldsymbol{x}} \Theta_{r}(\boldsymbol{x}) ; \quad \tilde{\eta}\left(p_{0}\right)=\int d x_{0} e^{i p_{0} x_{0}} \eta\left(x_{0}\right)
$$

and using Eqs. (11), (12) and (13), we get

$$
\begin{gathered}
\left\langle 0\left|\left[j^{0}\left(\Theta_{r} \eta\right), j^{0}(y)\right]\right| 0\right\rangle=\int d p \tilde{\Theta}_{r}(\boldsymbol{p})\left[e^{i p y} \tilde{\eta}\left(-p_{0}\right)-e^{-i p y} \tilde{\eta}\left(p_{0}\right)\right] \varrho(p) \\
+\int d p \tilde{\Theta}_{r}(\boldsymbol{p})\left[e^{i p y} \tilde{\eta}\left(-p_{0}\right)-e^{-i p y} \tilde{\eta}\left(p_{0}\right)\right] \boldsymbol{p}^{2} \varrho_{1}(p) .
\end{gathered}
$$

We will show now that, due to the presence of the factor $\boldsymbol{p}^{2}$ in the second term, this term drops when $r$ goes to infinity.

The $h$-function, which corresponds to the second term in Eq. (22) by Eq. (16), is

$$
h_{r}(\chi)=\int_{p_{0} \geqq 0} d p \boldsymbol{p}^{2} e^{i \boldsymbol{p} \cdot \boldsymbol{y}} \tilde{\Theta}_{r}(\boldsymbol{p})\left[e^{i p_{0} y_{0}} \tilde{\eta}\left(-p_{0}\right)-e^{-i p_{0} y_{0}} \tilde{\eta}\left(p_{0}\right)\right] \delta\left(p^{2}-\chi\right) \text {. }
$$

For every $\eta \in \mathscr{D}$ we have $\tilde{\eta} \in \mathscr{S}$ and it is easy to see that

$$
\frac{e^{i p_{0} y_{0}} \tilde{\eta}\left(-p_{0}\right)-e^{-i p_{0} y_{0}} \tilde{\eta}\left(p_{0}\right)}{2 p_{0}}=f\left(p_{0}^{2}\right) \in \mathscr{S}(R) \text { as function of } p_{0}^{2} .
$$

From Eq. (1) it follows that

$$
\tilde{\Theta}_{r}(\boldsymbol{p})=r^{3} \tilde{\Theta}(r \boldsymbol{p}) .
$$

Using Eqs. (23), (24) and (25), we get

$$
h_{r}(\chi)=\frac{1}{r^{2}} \int d \boldsymbol{p} \boldsymbol{p}^{2} e^{i \frac{\boldsymbol{p}}{\boldsymbol{r}} \cdot \boldsymbol{y}} \tilde{\Theta}(\boldsymbol{p}) f\left(\chi+\frac{\boldsymbol{p}^{2}}{r^{2}}\right) .
$$

Since $\hat{\varrho}_{1}(\chi)$ is a tempered measure, there exist an $N>0$ and a constant $K$ such that the following estimates for the absolute value of the second term in Eq. (22) hold [8]

$$
\begin{aligned}
\left|\int_{0}^{\infty} d \chi h_{r}(\chi) \hat{\varrho}_{1}(\chi)\right| & \leqq K \sup _{\chi>0}\left|(1+\chi)^{N} h_{r}(\chi)\right| \\
& \leqq \frac{K}{r^{2}} \int d \boldsymbol{p} \boldsymbol{p}^{2}|\tilde{\Theta}(\boldsymbol{p})| \sup _{\chi>0}\left|(1+\chi)^{N} f\left(\chi+\frac{\boldsymbol{p}^{2}}{r^{2}}\right)\right| \\
& \leqq \frac{K}{r^{2}} \sup _{\chi>0}\left|(1+\chi)^{N} f(\chi)\right| \int d \boldsymbol{p} \boldsymbol{p}^{2}|\tilde{\Theta}(\boldsymbol{p})|=\frac{K_{1}}{r^{2}} \underset{r \rightarrow \infty}{\longrightarrow} 0 .
\end{aligned}
$$

Where in the last equality we used the fact that $f \in \mathscr{S}$. 
Thus the second term in Eq. (22) vanishes when $r$ goes to infinity. We then have

$$
\begin{aligned}
& \lim _{r \rightarrow \infty}\left\langle 0\left|\left[j^{0}\left(\Theta_{r} \eta\right), j^{0}(y)\right]\right| 0\right\rangle \\
= & \lim _{r \rightarrow \infty} \int d p \tilde{\Theta}_{r}(\boldsymbol{p})\left[e^{i p y} \tilde{\eta}\left(-p_{0}\right)-e^{-i p y} \tilde{\eta}\left(p_{0}\right)\right] \varrho(p) .
\end{aligned}
$$

One part of Lemma 2 can be proven now. If we assume that $p^{\mu} \varrho(p)=0$ or $\varrho(p)=c \delta(p)$ we get that the expression in Eq. (27) vanishes for every $\eta \in \mathscr{D}(R)$ and every $y \in R^{4}$.

Let us assume now that the limit in Eq. (27) vanishes for every $\eta \in \mathscr{D}(R)$ and one arbitrary $y \in R^{4}$. The $h$-function which corresponds to Eq. (27) for finite $r$, by virtue of Eq. (16), is

$$
H_{r}(\chi)=\int_{p_{0} \geqq 0} d p e^{i \boldsymbol{p} \cdot \boldsymbol{y}} \tilde{\Theta}_{r}(\boldsymbol{p})\left[e^{i p_{0} y_{0}} \tilde{\eta}\left(-p_{0}\right)-e^{-i p_{0} y_{0}} \tilde{\eta}\left(p_{0}\right)\right] \delta\left(p^{2}-\chi\right) .
$$

Using Eqs. (24) and (25) we get

$$
\lim _{r \rightarrow \infty} H_{r}(\chi)=f(\chi) \int d \boldsymbol{p} \tilde{\Theta}(\boldsymbol{p})=(2 \pi)^{3} f(\chi) .
$$

Thus, the vanishing of the limit in Eq. (27) is equivalent to the statement that for every $f$, defined in Eq. (24), we have

$$
\int_{0}^{\infty} d \chi f(\chi) \hat{\varrho}(\chi)=0 .
$$

We claim that when $\eta\left(\chi_{0}\right)$ varies over $\mathscr{D}, f\left(p_{0}^{2}\right)$, as defined in Eq. (24), varies over a dense set in $\mathscr{S}$. In order to prove the claim, note that for every $f \in \mathscr{S}$ we can choose $\phi \in \mathscr{S}$ such that

$$
\frac{e^{i p_{0} y_{0}} \phi\left(-p_{0}\right)-e^{-i p_{0} y_{0}} \phi\left(p_{0}\right)}{2 p_{0}}=f\left(p_{0}^{2}\right) .
$$

The choice is

$$
\phi\left(p_{0}\right)=-e^{i p_{0} y_{0}} p_{0} f\left(p_{0}^{2}\right) .
$$

Thus, the proof that $\{f\}$ is dense in $\mathscr{S}$ reduces to the proof that for $\{\eta\}=\mathscr{D}$, the set $\{\tilde{\eta}\}$ is dense in $\mathscr{S}$. Since $\mathscr{D}$ is dense in $\mathscr{S}$ and the Fourier transfor is a continuous map of $\mathscr{S}$ onto itself, it follows that $\{\tilde{\eta}\}$ is dense in $\mathscr{S}$, thus $\{f\}$ is dense in $\mathscr{S}$ also.

From this conclusion and from Eq. (30), it follows that for every $h \in \mathscr{S}$

$$
\int_{0}^{\infty} d \chi h(\chi) \hat{\varrho}(\chi)=0 \text {. }
$$

Using the result, given after Eq. (16), we conclude that for every $g(p) \in \mathscr{S}\left(R^{4}-\{0\}\right)$

$$
\int d p g(p) \varrho(p)=0
$$


which is equivalent to the statement that the support of $\varrho$ is the point $p=0$. The general form of a measure with such a property is

$$
\varrho(p)=c \delta(p)
$$

which is equivalent to

$$
p^{\mu} \varrho(p)=0 .
$$

This concludes the proof of Lemma 2.

The proof of Theorem 2 is similar to that of Theorem 1 . We will now outline the proof of Theorem 2.

We start from a spectral representation for the tensor current two point function

$$
\left\langle 0\left|\theta^{\mu \nu}(x) \theta^{e \kappa}(y)\right| 0\right\rangle=\int d p e^{-i p(x-y)} \sigma^{\mu \nu e \kappa}(p) .
$$

The form of $\sigma^{\mu v e \kappa}(p)$, consistent with Eqs. (9) and (10), is

$$
\begin{aligned}
& \sigma^{\mu v \varrho \kappa}(p)=\sigma_{1}(p)\left[\left(p^{2}\right)^{2} g^{\mu v} g^{\varrho \kappa}-p^{2}\left(g^{\mu v} p^{\varrho} p^{\kappa}+g^{\varrho \kappa} p^{\mu} p^{v}\right)+p^{\mu} p^{v} p^{\varrho} p^{\kappa}\right] \\
& \quad+\sigma_{2}(p)\left[\left(p^{2}\right)^{2}\left(g^{\mu \varrho} g^{v \kappa}+g^{\mu \kappa} g^{v \varrho}\right)\right. \\
& \left.\quad-p^{2}\left(g^{\mu \varrho} p^{v} p^{\kappa}+g^{v \kappa} p^{\mu} p^{\sigma}+g^{\mu \kappa} p^{\varrho} p^{v}+g^{v \varrho} p^{\mu} p^{\kappa}\right)+2 p^{\mu} p^{v} p^{\varrho} p^{\kappa}\right]
\end{aligned}
$$

where $\sigma_{1}(p)$ and $\sigma_{2}(p)$ are Lorentz invariant tempered measures with support in $\bar{V}_{+}$. Let us define

$$
\sigma(p)=p^{2}\left[3 \sigma_{1}(p)+2 \sigma_{2}(p)\right]
$$

$\sigma(p)$ is also a Lorentz invariant tempered measure with support in $\bar{V}_{+}$.

Theorem 2 follows from two lemmas, whose proofs can be given along the same lines as the proofs of Lemma 1 and Lemma 2 respectively. The two lemmas are:

Lemma 3. The following two conditions are equivalent:

(i) $\theta_{\mu}{ }^{\mu}(y)=0$.

(ii) $\left(p^{\mu} p^{\nu}-p^{2} g^{\mu \nu}\right) \sigma(p)=0$.

Lemma 4. The following conditions are equivalent:

(i) Each of the three conditions (c)-(e) of Theorem 2.

(ii) $\left(p^{\mu} p^{\nu}-p^{2} g^{\mu \nu}\right) \sigma(p)=0$.

We will end with two consequences of Theorems 1 and 2 . Let $\mathscr{R}$ denote the algebra of unbounded field operators, i.e. polynomials in Wightman fields smeared with $\mathscr{D}\left(R^{4}\right)$ test functions. Using arguments similar to those of Chap. II we conclude that $\left\langle 0\left|\left[j^{0}(x), A\right]\right| 0\right\rangle$ in a $\mathscr{C}^{\infty}\left(R^{4}\right)$ function in $x$ for every $A \in \mathscr{R}$ which is (due to locality) a $\mathscr{D}\left(R^{3}\right)$ function in $\boldsymbol{x}$ for fixed $x^{0}$. The following is already a known result [10] which is a consequence of Theorem 1: 
Corollary 1. Under the assumptions of Theorem 1, the following condition

$$
\int d \boldsymbol{x}\left\langle 0\left|\left[j^{0}(x), A\right]\right| 0\right\rangle=0 \quad \text { for every } A \in \mathscr{R} \text { and one arbitrary } x^{0} \in R,
$$

yields

$$
\partial_{\mu} j^{\mu}(x)=0
$$

Similarly, one deduces from Theorem 2 a result which is Coleman's theorem for Conformal symmetry:

Corollary 2. Under the assumption of Theorem 2, each of the following conditions

1) $\int d \boldsymbol{x}\left\langle 0\left|\left[d^{0}(x), A\right]\right| 0\right\rangle=0 \quad$ for every $A \in \mathscr{R}$ and one arbitrary $x^{0} \in R$,

2) $\int d \boldsymbol{x}\left\langle 0\left|\left[\mathscr{K}^{00}(x), A\right]\right| 0\right\rangle=0$ for every $A \in \mathscr{R}$ and $x^{0}=0$,

3) $\int d \boldsymbol{x}\left\langle 0\left|\left[\mathscr{K}^{0 i}(x), A\right]\right| 0\right\rangle=0$ for every $A \in \mathscr{R}$, one $i=1,2,3$ and one arbitrary $x^{0} \in R$,

yields

and thus

$$
\theta_{\mu}{ }^{\mu}(x)=0
$$

$$
\partial_{\mu} d^{\mu}(x)=\partial_{\mu} \mathscr{K}^{\mu v}(x)=0
$$

Added Note: Parallely $\mathrm{H}$. Reeh treated the same problems, using slightly deferent technique [11].

Acknowledgements. The author wishes to express his gratitude to $\mathrm{H}$. Reeh for conversations and correspondence and to J. Slawny for his constant interest and helpful advice.

\section{References}

1. Gal-Ezer, E.: Nuovo Cimento A 10,682 (1972).

2. Streater,R.F., Wightman,A.S.: PCT, Spin \& Statistics and All That. New York: Benjamin 1964.

3. The original treatment, in which heuristic arguments are given, is Coleman, S.: J. Math. Phys. 7, 787 (1966). A review article, in which a rigorous formulation and proof is outlined and which contains previous references, is Orzalesi, G. A.: Rev. Mod. Phys. 42, 381 (1970). For a formulation and proof of a Coleman Theorem for the case of Conformal symmetry, see Bose, S. K., McGlinn, W. D.: Phys. Rev. D4, 342 (1971).

4. Schwartz, L.: Application of distributions to the theory of elementary particles in quantum mechanics. New York: Gordon and Breach, 1968.

5. $d^{\mu}(x)$ and $K^{\mu \nu}(x)$ are the currents generating the conformal group and $\theta^{\mu v}(x)$ is the "improved energy momentum tensor". See Callan, C. G., Jr., Coleman, S., Jackiw, R.: Ann. Phys. (N.Y.) 59, 42 (1970); Gell-Mann, M.: Proceedings of the 3rd Hawaii Topical Conference in Particle Physics, 1969 (Western Periodicals Co., Los Angeles).

6. Källen, G.: Helv. Phys. Acta 25, 417 (1952); Lehman, H.: Nuovo Cimento 11, 342 (1954). 
7. Wightman, A.S., Gårding, L.: Arkiv för Fysik 28, 129 (1965); Reeh,H.: Commun. math. Phys. 14, 315 (1969).

8. Gårding, L., Lions, J.L.: Functional analysis, Suppl. A1 Vol. XIV. Del Nuovo Cimento (1959).

9. Wightman, A.S.: Relativistic invariance and quantum mechanics. Suppl. A1 Vol. XIV Del Nuovo Cimento (1959).

10. Epstein, H., Hepp, K., Nuyts, J.: Quantum field theory seminar notes. Princeton (1966), unpublished preprint.

11. Reeh,H.: Coleman's Theorem, unpublished seminar talk, Max-Planck-Institut für Physik und Astrophysik.

Dr. Eldad Gal-Ezer

Dept. of Physics and Astronomy

Tel-Aviv University

Ramat Aviv/Israel 